nephron

Practice
Nephron 2018;140:94-98

DOI: $10.1159 / 000491558$
Received: May 28, 2018

Accepted after revision: June 25, 2018

Published online: July 13, 2018

\title{
Delayed Graft Function: The AKI of Kidney Transplantation
}

\author{
Roslyn B. Mannon
}

Division of Nephrology, Department of Medicine, University of Alabama at Birmingham, Birmingham, AL, USA

\section{Keywords}

Acute renal injury · Allograft · Chemokine · Outcomes ·

Transplantation

\section{Abstract}

With the growing wait list of individuals waiting for kidney transplantation, there has been renewed interest in the quality and function of donor organs. In particular, concern about the development of delayed allograft function (DGF) after transplantation continues to lead to the avoidance of donor organs offered to a transplant center. DGF is associated with worse short-and long-term outcomes and associated with higher rejection rates. There are no FDA-approved therapies to mitigate the ischemic injury that occurs. Risk factors include both donor and recipient characteristics, although their prediction is not precise. With new understanding about mechanisms of injury and new focus on the function of the deceased donor, there is opportunity to identify not only novel therapies to improve allograft function but to identify potential biomarkers of DGF. DGF remains a significant factor in impacting kidney transplant outcome, and finding biomarkers will assist in the development and ap- proval of novel agents to ameliorate early and later injury. This mini-review highlights our presentation at the 23rd International Conference on Advances in Critical Care Nephrology and UAB/UCSD O'Brien Center Acute Kidney Injury (AKI) Pre-Meeting.

(c) 2018 S. Karger AG, Basel

\section{Background}

While short-term kidney transplant outcomes are outstanding, long-term allograft survival continues to be a challenge. A significant proportion of kidney transplants rely on brain dead donors, although they have inferior outcomes compared to kidneys from living donors. In part, these outcomes relate to donor quality, as living do-

Contribution from the AKI and CRRT 2018 Symposium at the 23rd International Conference on Advances in Critical Care Nephrology, Manchester Grand Hyatt, San Diego, CA, USA, February 26 - March 1, 2018. This symposium was supported in part by the NIDDK funded University of Alabama at Birmingham-University of California San Diego O'Brien Center for Acute Kidney Injury Research (P30DK079337).

\section{KARGER}

(c) 2018 S. Karger AG, Basel

E-Mail karger@karger.com

www.karger.com/nef
Dr. Roslyn B. Mannon

Division of Nephrology, Department of Medicine

University of Alabama at Birmingham, 1900 University Boulevard, THT 643

Birmingham, AL 35294 (USA)

E-Mail rmannon@uabmc.edu 
nors are intensively screened for medical excellence and a lack of medical comorbidites, as well as ample renal function. Moreover, nephrectomy is a carefully timed procedure that limits both warm and cold ischemia. In contrast, deceased donors, while medically screened, are limited to known medical history, and the events leading to brain death may lead to functional impairment. Also, undermining deceased donor selection is the intense scarcity of organs available for transplant procedures and a waiting list that has been growing on a geometric basis over the last decade leading to less than optimal selection. Finally, brain death, in and of itself, induces an intense proinflammatory state, which may impact recipient immunity and graft function after kidney transplantation [1].

Delayed graft function (DGF) refers to the acute kidney injury that occurs in the first week of kidney transplantation, which necessitates dialysis intervention. DGF is associated with higher rates of acute cellular rejection and shorter graft survivals (reviewed in [2]). It often results in changes in maintenance immunosuppression therapy, specifically calcineurin inhibitor (CNI) use. There is a great variation in the incidence of DGF, and on average, it is $31 \%$ in US transplant centers. While prolonged cold ischemic time is associated with DGF, risk factors are primarily clinically based. We review the key features and risks for DGF, mechanistic insights, therapies, and possible biomarkers. We propose that the current clinical features are not specific enough to predict DGF post-transplantation and that better identification of the mechanisms of DGF are needed to guide therapeutic trials and identify biomarkers in this field.

\section{Clinical Implications of DGF}

The kidney donor profile index is a clinical measure of donor quality utilized in the kidney allocation scheme in US transplant centers and is based on age, height, weight, ethnicity, history of hypertension or diabetes, cause of death, serum creatinine, hepatitis $\mathrm{C}$ virus, and donation after cardiac death (DCD). Of deceased donors, the vast majority are brain dead while a smaller proportion are from DCD donors. DGF rate is about $30.8 \%$ in US deceased donors [3] which is significantly higher in DCDs (45-55.1\%) [4]. The incidence of DGF is dependent not only on the length of cold ischemia to the organ but also on the extent of warm ischemic injury, which tends to be lengthier in DCD by the nature of the induction of cardiac death, as well as other factors such as the kidney donor profile index. Alternatively, traumatic brain death may be accompanied by a syndrome of thrombotic mi- croangiopathy that can be additive to kidney injury and may be accelerated or supported by the use of CNIs and/ or mammalian target of rapamycin (mTOR) inhibitors used for maintenance therapy (reviewed in [2]).

The development of DGF is associated with worse baseline kidney transplant function and shorter graft survival periods, which are anywhere from 3 to 5 year shorter graft half-life as demonstrated in a recent single-center analysis [4]. Poor function in the immediate postoperative period necessitates the use of dialysis from anywhere from days to months, which adds a significant cost impact to patient management [5]; it also complicates post-transplant management as an outpatient. Immunosuppressive therapy, particularly the maintenance use of CNIs, which in and of themselves is associated with acute nephrotoxicity, is frequently minimized or not utilized, suggesting that one mechanism is linking DGF to early rejection, that is, under-immunosuppression. There has not been clearcut evidence about the impact of induction antibody therapy, although North American transplant centers typically use rabbit anti-thymocyte globulin to minimize CNI dosing early on, while across the globe, anti-CD25 monoclonal therapy is the primary induction agent.

\section{Mechanisms of AKI/DGF}

A thorough and in-depth discussion can be found in [2]. Much like AKI seen in native kidneys, DGF is associated with innate immune activation, with complement activation and released damage-associated molecular patterns such as hypomethylated DNA, hyaluronic acid, HMGB1, heparin sulfate, fibrinogen, and heat shock proteins. These molecules transmit their signals through tolllike receptors particularly in the proximal tubular epithelium [6]. Engagement of toll like receptors (TLR) activates the NFkB pathway through the adapter protein MyD88, and this leads to the induction of inflammatory cytokines such as interleukin 1 (IL-1), interleukin 6 (IL6), tumor necrosis factor alpha (TNFa), and interferon beta (IFN $\beta$ ). Furthermore, TLR activation directly stimulates macrophage inducible nitric oxide synthaste (iNOS) as well as the activation of the NADPH oxidase system. Finally, these interactions can lead to the activation of interstitial dendritic cells with upregulation of CCR7 promoting cell migration to secondary lymph nodes, upregulation of expression of costimulatory molecules (CD80/ B7-1, CD86/B7-2), and production of IL-12, the latter leading to Th1 cell differentiation. This latter event in particular is essential in the priming role of antigen-reactive $\mathrm{T}$-cell responses including responses to allografts and leads to crosstalk with the adaptive immune response. 
Table 1. Risk factors for the development of delayed graft function, with characteristics (shown in bold) identified in multiple studies (adapted from [18])

\begin{tabular}{ll}
\hline Donor-related & Recipient-related \\
\hline Female gender & Male gender \\
Increasing age & Black race \\
Body mass index & Body mass index \\
Deceased vs. living donation & Previous transplant \\
Donation after cardiac death & Diabetes \\
Increasing donor serum creatinine & Pre-transplant dialysis versus none \\
Cause of death (anoxia vs. cerebrovascular) & Duration and type of dialysis \\
Duration of intensive care stay & Residual diuresis \\
Duration of brain death & Pre-transplant transfusion \\
Diabetes & Pre-transplant blood pressure \\
History of hypertension & Pre-transplant lipid profile \\
Graft atherosclerosis & Cardiac function \\
\hline Preservation & Transplant-related \\
\hline Cold ischemic time (CIT) & Sensitization \\
Warm ischemic time & Human Leukocyte Antigen (HLA) mismatches \\
& ABO Blood group incompatible \\
\hline
\end{tabular}

Thus, there is a room for recipient immune activation and acute rejection of the graft.

A number of groups have examined the role of innate immune molecules in preclinical in avivo models of AKI. Such work has identified complement activation as a key feature of ischemic kidney injury [2]. Such studies have found upregulation of interleukin-6 levels and hepatocyte growth factor, and disruption of such pathways has been associated with mitigation of injury histologically and functionally. However, preclinical studies have not been entirely accurate in predicting responses in human treatment trials; for example, the infusion of superoxide dismutase, a free radical scavenger, blockade of ICAM-1, or treatment with pentoxyfyline to inhibit TNFa have not been consistent in terms of mitigating DGF as they have been in rodents. Such studies highlight the differences in rodent and human inflammatory responses and the genetic diversity inherent in man that influence ischemic innate injury and alloimmune responses.

\section{Risk Factors for DGF}

There are multiple studies identifying risk factors for DGF. These are summarized in Table 1. With the complexity and interplay of both donor and recipient factors, a number of groups have created risk factor prediction models using large data registries that are publically available. The most widely used, with about $70 \%$ accuracy, is the Irish risk calculator This calculator is based on 20 independent recipient- and donor-related risk factors and identified the most significant factors associated as cold ischemic time, donor terminal creatinine, donor body mass index, DCD, and donor age [7]. However, there is still disagreement about its ability to predict clinical events, in part, due to the limitations of granularity in registry data.

\section{Are there Relevant Biomarkers?}

The study of such mechanisms not only paves the way for therapeutic innovation but also allows for identifying biomarkers of irreparable injury. Moreover, the imprecise nature of both donor and recipient clinical factors to predict DGF development indicates an unmet need of biomarkers of injury. To this end, according to a prospective study involving a number of US transplant centers, higher urinary NGAL and IL-18 levels in recipients on the first post-transplant day were associated with higher DGF rates and poor allograft function at 12 months post transplantation [8]. Similarly, higher BDD urinary NGAL concentrations were associated with DGF with the highest versus lowest NGAL tertile relative risk of 1.21 (95\% CI 1.02-1.43) [9]. Recent studies by Pianta et al. [10] have identified urinary expression of clusterin, IL-18, and KIM-1 as well as urinary tissue inhibitor of metalloproteinases- 2 and insulin-like growth factor-binding protein $7[10,11]$ as an addition to clinical features in predicting early DGF. These studies focus on recipient expression and not on that of the deceased donor. 


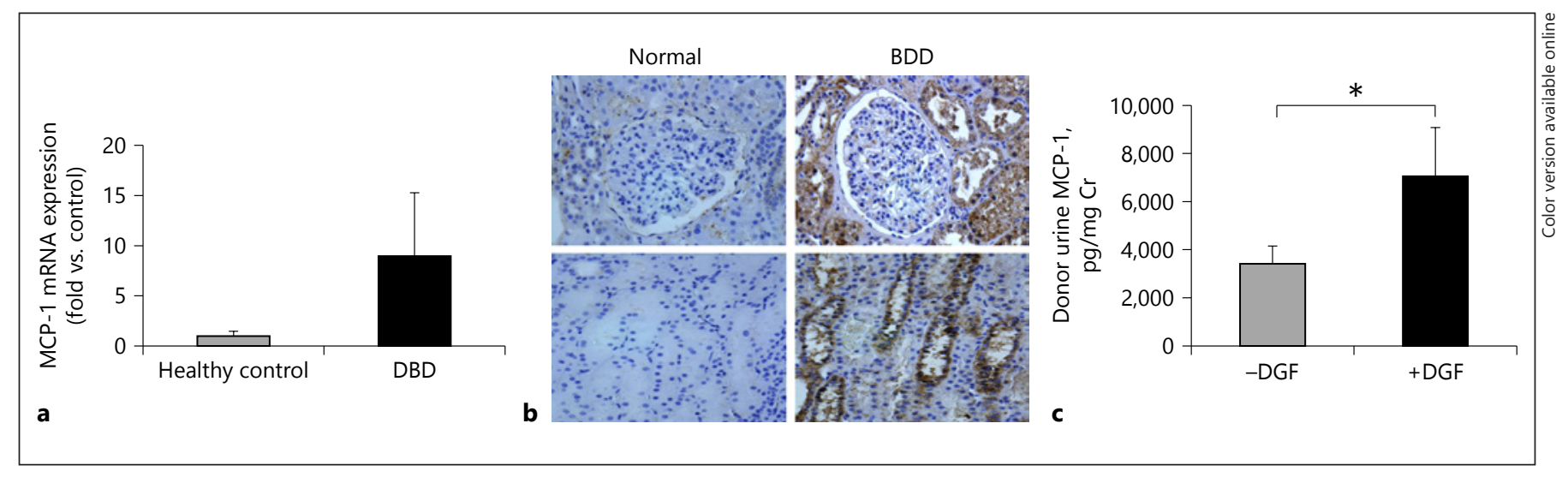

Fig. 1. Monocyte chemoattractant protein-1 (MCP-1) expression in $\mathrm{BD}$ donor serum, urine, and kidney biopsies. a Gene expression of MCP-1 in BDD kidney biopsies $(n=29)$ was upregulated compared to normal, healthy controls $(n=4)$, although this was not statistically significant $(p=0.649)$. Values are fold induction compared to healthy controls, mean \pm SEM. b Representative immu-

We have recently initiated a prospective study of brain dead donors (BDD) at our donor center with the goal of correlating donor management and clinical features, with possible biomarkers of DGF in recipients. As elevated levels of recipient urinary monocyte chemoattractant protein-1 (MCP-1), which is a powerful chemoattractant for monocytes and macrophages, have been associated with interstitial macrophage infiltration, interstitial fibrosis and inflammation, and DGF [12], we first assessed levels of MCP-1 in the donor. While we found no differences in systemic levels of MCP-1, MCP-1 was elevated in both the renal parenchyma and urine, and was associated with DGF (Fig. 1). Along these lines, Mansour et al. have shown that DBD urine MCP-1 is associated with AKI, which occurred only in $9 \%$ of recipients studied, although sample collection was inconsistent across donor hospitals and facilities [13]. These studies highlight the potential of BDD biosamples as adjunct to more precisely predicting those at risk for DGF.

\section{Treatment}

There have been investigations in both deceased donors and recipients to mitigate the ischemic injury, but to date, there is no FDA-approved therapy. Based on work by Moers et al. [14], hypothermic pump perfusion is utilized by organ procurement centers to minimize DGF. Interventions in recipients have not been entirely successful in mitigation DGF such as dopamine and superoxide dismutase infusions, fenoldapam, and anti-ICAM1 nohistochemical staining for MCP-1 in kidney biopsies from BDD and healthy controls (upper panels 100×, lower panels 200×). Expression was localized to tubular epithelium. c Urine MCP-1 levels stratified by recipients with delayed graft function (DGF; +DGF) and those without (-DGF). DGF was associated with significantly higher urine levels in $\mathrm{BDD}(* p=0.0498)$.

treatment (reviewed in [2]). As yet unreported phase III studies that include the use of anti-C5 antibody and silencing RNA to p53 to limit cellular apoptosis. There are single-center, phase I studies that are encouraging in terms of limiting ischemic renal injury through the inhibition of IL- 6 and HGF. More definitive clinical studies in large-volume transplant centers are currently underway.

Donor management investigation has been less well studied but may be a critical opportunity to improve organ quality. In this landmark study in donor management, Niemann demonstrated that modest donor hypothermia significantly reduced DGF rates from 39 to $28 \%$ [15]. While these differences are modest, they do demonstrate improvement in transplanted organs and could translate into more usable organs and organs that have less damage to begin with. Critically in this work is the complex process of experimental management whose risks and benefits may extend beyond the sole purpose of better kidney function, and further complicate the process of organ allocation, and in particular utilization, when recipient informed consent is required. Such complexities, which are a major hurdle to donor management therapies was the focus of the 2017 Institute of Medicine report [16].

\section{The Future}

It is important to reiterate that clinical features of the brain dead donor and renal function are not precise predictors of the development of DGF. Husain and col- 
leagues explored the clinical characteristics of unilateral kidney transplant recipients stratified by the reason that the partner kidney was discarded. They demonstrated that the rate of DGF was $40 \%$ when there was extended ischemia, 38\% when donor function was felt to be insufficient, and $37 \%$ when a kidney was discarded due to concerns of donor biopsy pathology [17]. This indicates the imprecise nature of the assessment of clinical outcome following kidney implantation and calls for the need for developing new tools to predict later function and transplant excellence.

\section{Disclosure Statement}

The author has no conflicts of interest to declare.

\section{References}

1 Watts RP, Thom O, Fraser JF: Inflammatory signalling associated with brain dead organ donation: from brain injury to brain stem death and posttransplant ischaemia reperfusion injury. J Transplant 2013;2013:521369.

2 Siedlecki A, Irish W, Brennan DC: Delayed graft function in the kidney transplant. Am J Transplant 2011;11:2279-2296.

3 Wang CJ, Wetmore JB, Israni AK: Old versus new: progress in reaching the goals of the new kidney allocation system. Human Immunol 2017;78:9-15.

4 Zens TJ, Danobeitia JS, Leverson G, Chlebeck PJ, Zitur LJ, Redfield RR, et al: The impact of kidney donor profile index on delayed graft function and transplant outcomes: A singlecenter analysis. Clin Transplant 2018;32: e13190.

5 Buchanan P, Schnitzler M, Axelrod D, Salvaggio $P$ : The clinical and financial burden of early dialysis after deceased donor kidney transplantation. J Nephrol Therapeutic 2011;S4: 001.

6 Rosin DL, Okusa MD: Dangers within: DAMP responses to damage and cell death in kidney disease. J Am Soc Nephrol 2011;22: 416-425.

7 Irish WD, Ilsley JN, Schnitzler MA, Feng S, Brennan DC: A risk prediction model for delayed graft function in the current era of de- ceased donor renal transplantation. Am J Transplant 2010;10:2279-2286.

8 Hall IE, Yarlagadda SG, Coca SG, Wang Z, Doshi M, Devarajan P, et al: IL-18 and urinary NGAL predict dialysis and graft recovery after kidney transplantation. J Am Soc Nephrol 2010;21:189-197.

9 Reese PP, Hall IE, Weng FL, Schroppel B, Doshi MD, Hasz RD, et al: Associations between deceased-donor urine injury biomarkers and kidney transplant outcomes. J Am Soc Nephrol 2016;27:1534-1543.

10 Pianta TJ, Peake PW, Pickering JW, Kelleher M, Buckley NA, Endre ZH: Evaluation of biomarkers of cell cycle arrest and inflammation in prediction of dialysis or recovery after kidney transplantation. Transpl Int 2015;28: 1392-1404.

11 Pianta TJ, Peake PW, Pickering JW, Kelleher M, Buckley NA, Endre ZH: Clusterin in kidney transplantation: novel biomarkers versus serum creatinine for early prediction of delayed graft function. Transplantation 2015; 99:171-179.

12 Ho J, Wiebe C, Gibson IW, HombachKlonisch S, Gao A, Rigatto C, et al: Elevated urinary CCL2: $\mathrm{Cr}$ at 6 months is associated with renal allograft interstitial fibrosis and inflammation at 24 months. Transplantation 2014;98:39-46.
13 Mansour SG, Puthumana J, Reese PP, Hall IE, Doshi MD, Weng FL, et al: Associations between deceased-donor uMCP-1 and kidney transplant outcomes. Kidney Int Rep 2017;2:749-758.

14 Moers C, Smits JM, Maathuis MH, Treckmann J, van Gelder F, Napieralski BP, et al: Machine perfusion or cold storage in deceased-donor kidney transplantation. $\mathrm{N}$ Engl J Med 2009;360:7-19.

15 Niemann CU, Feiner J, Swain S, Bunting S, Friedman M, Crutchfield M, et al: Therapeutic hypothermia in deceased organ donors and kidney-graft function. N Engl J Med 2015;373:405-414.

16 National Academies of Sciences E, Medicine Opportunities for Organ Donor Intervention Research: Saving Lives by Improving the Quality and Quantity of Organs for Transplantation. James FC, Sarah D, Catharyn TL, editors. Washington, The National Academies Press, 2017.

17 Husain SA, Chiles MC, Lee S, Pastan SO, Patzer RE, Tanriover B, et al: Characteristics and performance of unilateral kidney transplants from deceasedd. Clin J Am Soc Nephrol 2018;13:118-127.

18 Nashan B, Abbud-Filho M, Citterio F: Prediction, prevention, and management of delayed graft function: where are we now? Clin Transplant 2016;30:1198-1208. 